Europhys. Lett., 50 (2), pp. 135-141 (2000)

\title{
Polycontinuous morphologies and interwoven helical networks
}

\author{
S. T. HydE and S. RAMSDEN \\ Department of Applied Mathematics, Research School of Physical Sciences \\ Australian National University, Canberra, ACT 0200, Australia
}

(received 25 October 1999; accepted in final form 27 January 2000)

PACS. 02.40.Sf - Manifolds and cell complexes.

PACS. 61.25.Em - Molecular liquids.

PACS. $61.30 . \mathrm{Cz}$ - Theory and models of liquid crystal structure.

\begin{abstract}
We describe a construction procedure for polycontinuous structures, giving generalisations of bicontinuous morphologies to more than two equivalent, continuous and interwoven sub-volumes. The construction gives helical windings of disjoint graphs on triply periodic hyperbolic surfaces, whose universal cover in the hyperbolic plane consists of packed, parallel trees. The simplest tri-, quadra- and octa-continuous morphologies consist of three $(8,3)-c$, four $(10,3)-a$ and eight $(10,3)-a$ interwoven networks, respectively. The quadraand octa-continuous cases are chiral. A novel chiral bicontinuous structure is also derived, closely related to the well-known cubic gyroid mesophase.
\end{abstract}

Introduction. - The concept of a bicontinuous morphology has proven to be a useful one in understanding structures at various length scales in a variety of (sometimes partially) condensed atomic, molecular and macromolecular systems [1-3]. These phases are now recognised in a range of natural and synthetic atomic frameworks, lyotropic and thermotropic liquid crystalline systems, and block copolymers [4]. A useful model of bicontinuous crystalline (meso)phases is a single-sheeted crystalline dividing surface that is close to a triply periodic minimal surface or TPMS (an ordered "sponge"). The most commonly encountered surfaces in liquid crystalline mesophases are the three genus-three cubic TPMS: the $P, D$ and gyroid $(G)$ surfaces. Alternatively, the surface topology can be characterised by its "labyrinth" graphs, that describe the channel architecture carved out by the hyperbolic surface (fig. 1). The self-assembly process that leads to these morphologies in molecular systems has been described in detail elsewhere [5-7]. The stability of the $P, D$ and $G$ surfaces in molecular assemblies is likely to be due to their "quasi-homogeneous" geometries, with minimal variations of Gaussian curvature and channel radii compared with other known minimal surfaces.

Polycontinuous morphologies. - Polycontinuous structures consist of identical ( $n$ say) interwoven $3 d$ domains. Bicontinuous forms can be constructed by aggregation of di-block rods with mutually immiscible blocks (surfactants, copolymers,...). Polycontinuous morphologies result from aggregation of star-shaped rods, each consisting of $n$-arms, where the number of domains may exceed two. Tri-, quadra-, ... continuous morphologies cannot be trivial (C) EDP Sciences 


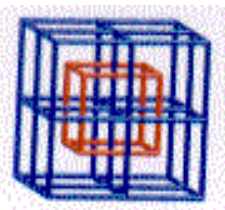

(a)

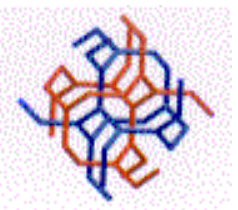

(b)

Fig. 1 - Pair of interwoven labyrinth graphs defining the channels of (a) the $P$ and (b) the $G$ surfaces.

extensions of the surfaces that separate immiscible domains in bicontinuous phases, since neighbouring star-shaped rods can only be stacked along one-dimensional lines, so that the star centres lie on graphs rather than surfaces. The simplest $3 d$ star-aggregates can be inferred from planar three- and four-connected networks. Stacks of Y-shaped stars can be placed endto-end to form a hexagonal honeycomb network; X-shaped stars can form a square honeycomb (fig. 2). More generally, the node density can be "tuned" by twisting the stars along the vertical axis. The tips of the stars trace helical wrappings on cylindrical surfaces, so that each cylinder resembles a barber-pole (fig. 2). These $2 d$ Euclidean patterns will be curved, via insertion of disclination networks, to give polycontinuous morphologies.

The $2 d$ Euclidean nature of these wrappings follows from the vanishing Gaussian curvature of the rods. Unglue each rod along a seam that runs along its length, to form a flat sheet. The helices unfold, to form a sheaf of parallel "diagonals". Reversing that construction allows any helical wrapping to be generated from decoration of a $\{4,4\}$ tiling of $E^{2}$, the Euclidean plane. (Denote a tiling by its Schläfli symbol, $\{n, z\}$, where the first entry is the ring size, and the second the number of edges about each vertex). The helical pitch is dependent on the angle the helix subtends with the edges of the $\{4,4\}$ network, or the vertex $(x, y)$ of the $\{4,4\}$ network closest to the origin that is visited by the helix (where the origin is located at $x$

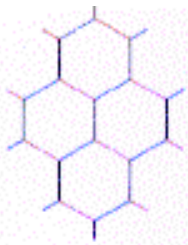

(a)

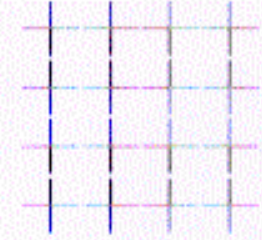

(b)

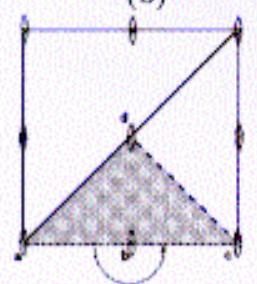

(e)

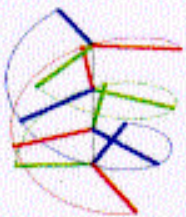

(c)

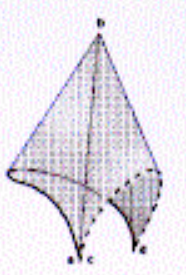

(f)

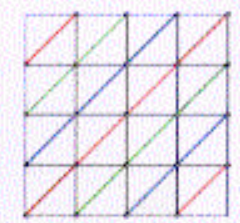

(d)

Fig. 2 - Planar end-to-end arrangements of (a) 3- and (b) 4-armed stars. (c) Twisted stacking of 3 -armed stars, forming helices. (d) Universal cover of $(1,1)$ helical windings on a cylinder in $E^{2}$, of orbifold symmetry $2 * 22$ (ignoring the colouring). (e) Single unit of (d), with mirrors along $a d$ and $c d$, and two-fold rotational symmetry sites at $a, b, c$ and $d$. (f) Compactified $2 * 22$ orbifold from (e), formed by joining edges linked by the arrow. The orbifold is bounded by mirrors, with an order 2 cone point at $d$, formed from (d) by identification of edges $a b$ and $c b$. 
and $y$ directions lie along the curved and straight principal directions of the rod, respectively, and the circumference of the rods is set to unity). Choose the simplest symmetric helical patterns, with pitch $(1,0)$ and $(1,1)$ (fig. 2(d)). (Other examples are canvassed in [8].) The symmetries of these planar patterns are $p 2 \mathrm{~mm}$ and $c 2 \mathrm{~mm}$, or in the more transparent orbifold nomenclature of Conway [9], $* 2222$ and $2 * 22\left({ }^{1}\right)$.

In order to generate polycontinuous morphologies, we generalise these barber-pole patterns to wrappings on sponges embedded in $E^{3}$. To ensure quasi-homogeneity of the final patterns, we consider only the most symmetric genus-three TPMS. We analyse first the wrappings in $H^{2}$, the universal cover of sponges. The Euclidean $2 * 22$ orbifold can be "edited" [10] to give a related two-dimensional symmetry group in $H^{2}$. The simplest hyperbolic analogue of the kaleidoscopic $* 2222$ case is $* 2223$, likewise $2 * 23$ is the analogue of the $2 * 22$ orbifold. In slightly more generality, $2 * 2 i, 22 * i$ and $222 * i$ orbifolds are possible symmetry groups in $H^{2}$, where $i$ exceeds two [9]. That editing process is accomplished by insertion of disclinations on the helical pattern, with disclinations located at all common vertices $(x, y)$. For sufficiently low disclination densities the (Clifford) parallel helical lines form infinite arrays of non-intersecting trees in $H^{2}$. To embed the network in $E^{3}$, a transformation from the forested $H^{2}$ to the triply periodic sponge is imposed, via a tiling of $H^{2}$. Judicious selection of the forest results in a $3 d$ helical winding of the sponge containing a number of intertwined helices.

Sadoc and Charvolin have demonstrated the use of the $\{4,6\}$ tiling as a map for the $P$, $D$ and $G$ triply periodic minimal surfaces [11]. (Note that although the global embeddings of the $P, D$ and $G$ surfaces differ in $E^{3}$, their Gauss maps are identical.) Here we adopt a slightly more general algorithm for the underlying map from $H^{2}$ to the minimal surface in $E^{3}$. Since the surfaces are minimal, the Gauss map is conformal, except at isolated flat points on the surfaces. It is necessarily branched at the surfaces' flat points, of order $b$. Further, the mapping of simpler "regular" three-periodic minimal surfaces (defined in ref. [12]) is very symmetric: the orbifolds are kaleidoscopic. Fundamental domains for the Gauss maps of simpler TPMS (derived elsewhere [12]) can be used to form maps of the surfaces in $H^{2}$ as follows. Domain edges are geodesics, we demand that they are also geodesics in $H^{2}$. Vertex angles in $H^{2}$ are shrunk by a factor of $\frac{1}{b+1}$ relative to those on the Gauss map, where $b$ denotes the branch point order of the vertex on the Gauss map. That algorithm applied to the simplest TPMS, the genus-three cubic $P / D / G$ family of triply periodic minimal surfaces and the genus-three hexagonal $H$ surface, produces the "kaleidoscopic nets" of symmetries $* 246$ and $* 2226$, shown in fig. 3 . (Note that the $* 2226$ pattern is in fact a one-parameter family of nets, corresponding to the variable axial ratio of the hexagonal surface. We choose herefor convenience only - the most symmetric member of that family in $H^{2}$, that is generated from the more symmetric 24(12) orbifold.) Those kaleidoscopic nets of $H^{2}$ contain simple Platonic sub-graphs, related to the Euclidean $\{4,4\}$ network: the $P / D / G$ net contains the $\{4,6\}$ graph, the $H$ net contains $\{4,12\}$. To obtain the hyperbolic analogues of the helical $(1,0)$ and $(1,1)$ patterns, insert networks of (negative) disclinations at vertices of the original $\{4,4\}$ tiling. Those disclinations increase connectivity of the original (2-connected) unfolded helical diagonals, resulting in 3- and 6-connected trees in $H^{2}$ superimposed on the $\{4,6\}$ and $\{4,12\}$ nets, respectively, shown in fig. $3\left(^{2}\right)$. Finally, the patterns in $H^{2}$ are wrapped (with

$\left({ }^{1}\right)$ A generic 2D symmetry group containing rotation and reflection symmetries is labelled $a b \ldots c * d e \ldots f$. Entries $a, \ldots$ preceding the asterisk denote sites of $a$-fold rotational symmetry; entries $d, \ldots$ conjunctions of rotational symmetry lying on mirrors intersecting at $\frac{2 \pi}{d}$. (If the symmetry order exceeds single digits, the entry is contained between parentheses, e.g. 36(12) denotes an orbifold bounded by 3-, 6- and 12-fold rotational symmetries.) Orbifolds bounded exclusively by mirrors (*de...) are called "kaleidoscopic".

$\left({ }^{2}\right)$ The tree morphologies can be inferred by calculating the diagonal edge lengths, which exceed the values required to form closed loops. 


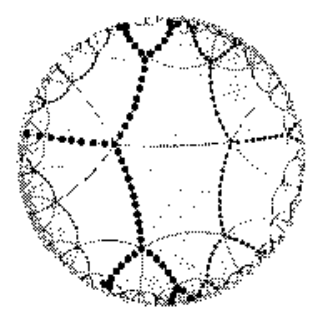

(a)

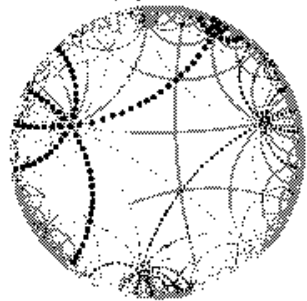

(d)

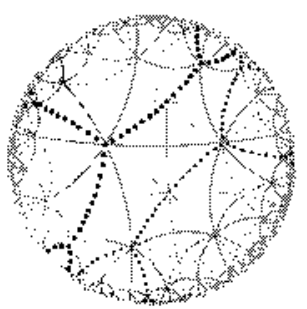

(b)

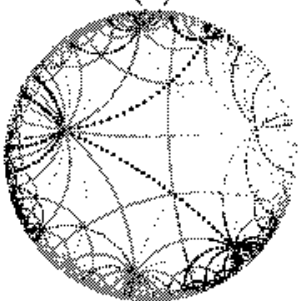

(e)

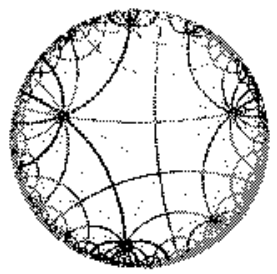

(c)

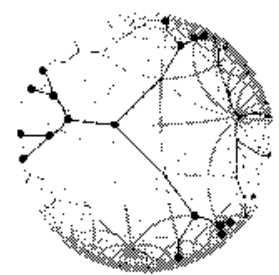

(f)

Fig. 3 - Packed 3-connected trees of edge length (a) $\cosh ^{-1}(3)$ and (b) $\cosh ^{-1}(5)$ superposed on the $\{4,6\}$ tiling. (c), (d) The corresponding trees superposed on the Platonic $\{4,12\}$ tiling. (e) Less symmetric 6 -connected trees on $\{4,12\}$; (f) 3 -connected trees on $\{4,12\}$ formed by replacing each vertex of the previous pattern with a Y-shaped star of edges.

some distortion of the metric, required to embed $H^{2}$ in $E^{3}$ ) according to the gluings needed to form the $P / D / G$ and $H$ surfaces in $E^{3}$. That process has been described for the $P / D / G$ family in [6], and is generalised elsewhere [8]. The wrappings result in multiple disjoint networks in $E^{3}$, whose translational symmetries are generally subgroups of their underlying surfaces [8].

The topology of those networks is dependent on the helical pitch, and the embedding process into $E^{3}$. Some trees form zero pitch helices on the surfaces in $E^{3}$, resulting in either $3 d$ lattices of finite ("molecular") graphs, or lattices of planar graphs. These examples, while interesting and of possible relevance to atomic and molecular structures [8], are oblique to the goal of this paper.

A number of polycontinuous examples result, listed in table I. Interwoven $3 d$ graphs result from the 3 -connected trees of edge-length $\cosh ^{-1}(3)$ and $\cosh ^{-1}(5)$ overlayed on the $\{4,6\}$ net, followed by embedding in $E^{3}$ via the $D$ and $P$ surfaces, respectively. In both cases, the

TABLE I - Hyperbolic symmetries of z-connected trees on kaleidoscopic nets characteristic of the genus three cubic $P / D / G$ and hexagonal $H$ triply periodic minimal surfaces, and the $3 d$ space group symmetries of resulting helical networks on these surfaces. The net symbols are either those of Wells for $3 d$ infinite graphs [13].

\begin{tabular}{ccccccc}
\hline $\begin{array}{c}\text { Edge } \\
\text { length }\end{array}$ & $\begin{array}{c}H^{2} \\
\text { symmetry }\end{array}$ & Surface & Nets & $\begin{array}{c}E^{3} \\
\text { symmetry } \\
\text { of each net }\end{array}$ & $\begin{array}{c}\text { Number } \\
\text { of disjoint } \\
\text { nets }\end{array}$ & $\begin{array}{c}E^{3} \\
\text { symmetry }\end{array}$ \\
\hline $\cosh ^{-1}(3)$ & $* 2223$ & $D$ & $\{10,3\}-a$ & $I 4_{1} 32$ & 4 & $P 4_{2} 32$ \\
$\cosh ^{-1}(5)$ & $2 * 23$ & $P$ & $\{10,3\}-a$ & $I 4_{1} 32$ & 8 & $I 432$ \\
$\cosh ^{-1}(3)$ & $* 2223$ & $G$ & $\{10,3\}-a($ distorted $)$ & $P 4_{3} 32$ & 2 & $I 4_{1} 32$ \\
$\cosh ^{-1}\left(\frac{\sqrt{3}+1}{\sqrt{3}-1}\right)$ & $22 * 3$ & $H$ & $\{8,3\}-c$ & $P 6_{3} / m m c$ & 3 & $P 6_{3} / m c m$ \\
\hline
\end{tabular}




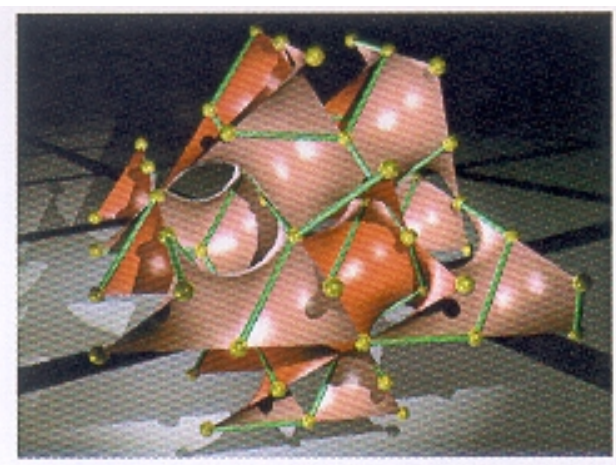

(a)

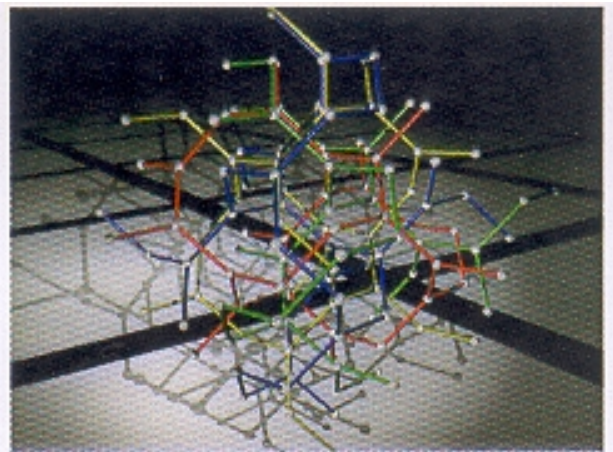

(b)

Fig. 4 - (a) Embedding of the 3-connected trees shown in fig. 6 in the $D$ surface, resulting in (b) four discrete interwoven graphs. The edges lie on straight lines of the surface, and each discrete graph is a right-handed $(10,3)-a$ graph $\left(Y *_{+}\right)$.

windings are collections of chiral $\{10,3\}-a$ nets (to use the nomenclature of Wells [13]), with vertices on the $Y *$ lattice complex of the $I a 3 d$ space group. Four identical enantiomers of the $\{10,3\}-a$ network wrap around the $D$ surface (figs. 4), and eight wrap the $P$ surface. These wrappings define labyrinth graphs of chiral quadra- and octa-continuous structures, respectively (figs. 5). Both helical patterns form "dogs-leg" motifs, with graph edges twisting about each catenoidal channel of the minimal surface. Four discrete graphs wind each channel of the $P$ surface and three wrap around channels of the $D$ surface (fig. 6).

The 3 -connected tree of edge length $\cosh ^{-1}(3)$ can also be embedded in the gyroid to form a pair of chiral (geometrically distorted) $\{10,3\}-a$ networks. If the curved edges are straightened in $E^{3}$ (fixing the vertex positions on the surface) the angles between straight edges are equal to the tetrahedral angle, $\arccos \left(-\frac{1}{3}\right)$, resulting in a pair of cubic $\{10,3\}-a$ graphs of identical handedness, each a subgraph of the diamond graph (figs. 7). This novel chiral bicontinuous pattern differs from that of the gyroid, that partitions space into a pair of $\{10,3\}-a$ graphs of opposite handedness.

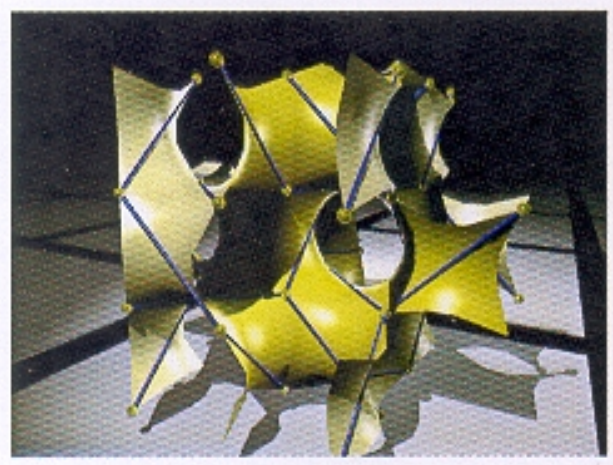

(a)

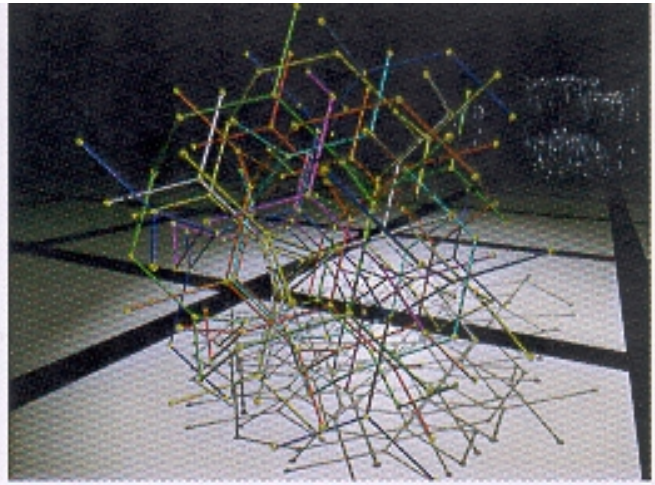

(b)

Fig. 5 - (a) Embedding of the 3 -connected trees shown in fig. 3 in the $P$ surface; they result in (b) eight right-handed interwoven cubic $(10,3)-a$ graphs. 


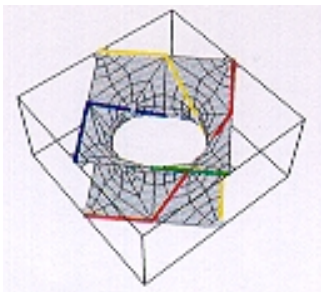

(a)

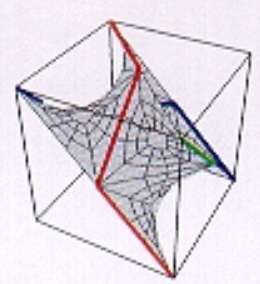

(b)

Fig. 6 - Winding of edges around catenoidal channels in the (a) $P$ and (b) $D$ surfaces. Notice the dogs-leg motifs in both cases. The $P$ channels are wrapped by four such legs, the $D$ by three.

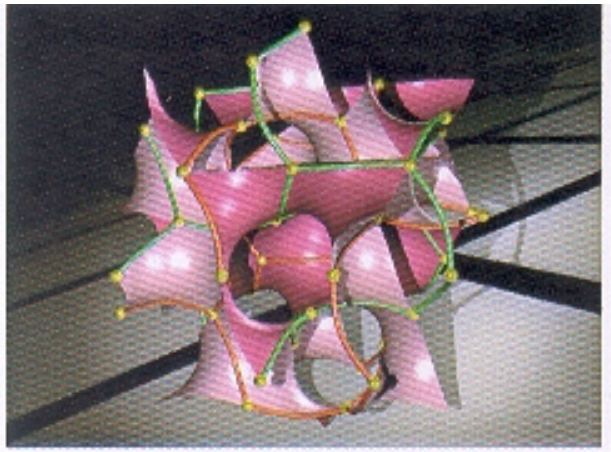

(a)

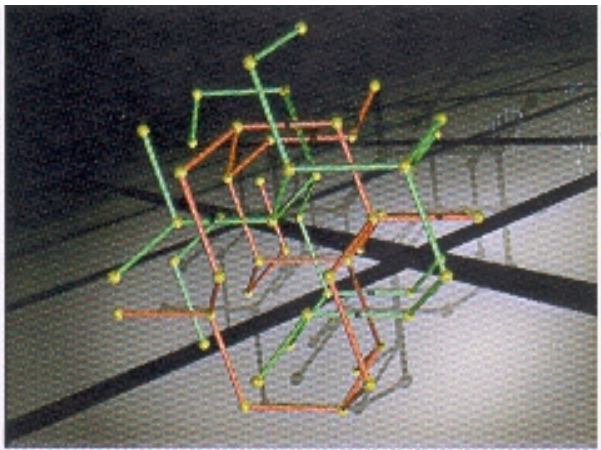

(b)

Fig. 7 - (a) Embedding of the 3-connected trees shown in fig. 3 in the $G$ surface, resulting in (b) two right-handed interwoven graphs. The graphs are slightly distorted versions of the cubic $(10,3)-a$ examples shown in the previous figures.

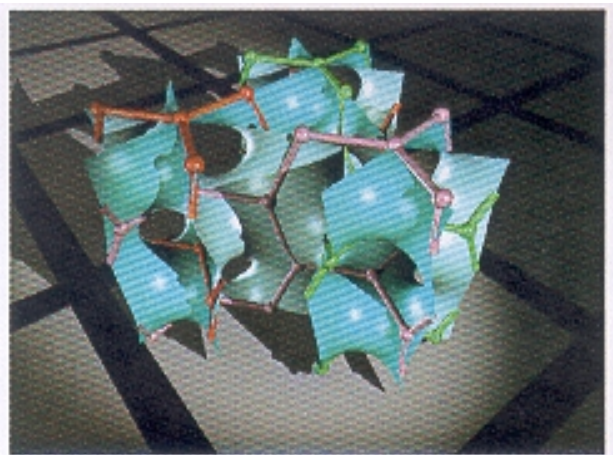

(a)

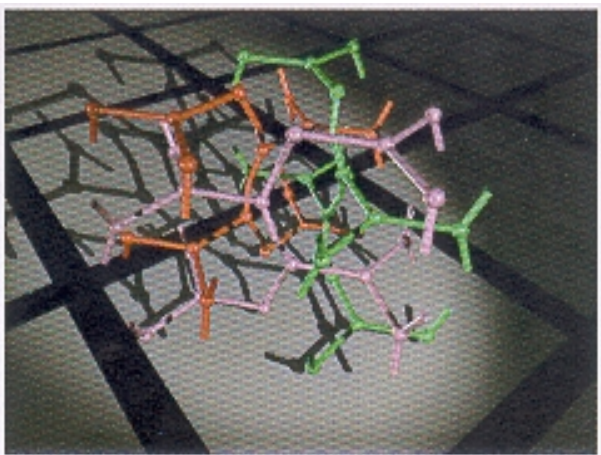

(b)

Fig. 8 - (a) Embedding of the 3-connected trees shown in fig. 3 in the $H$ surface, resulting in (b) three interwoven $(8,3)-c$ hexagonal graphs. 
These cubic embeddings do not admit morphologies with odd numbers of graphs. To generate a trio of identical 3-connected nets, a hexagonal sponge, the $H$ surface, is invoked. Choose the dogs-leg motif on the $H$ surface. Tricontinuous achiral structures result from embedding of 3 -connected trees of $2 d$ symmetry $22 * 3$ on the $\{4,12\}$ net of the $H$ surface, forming the $\{8,3\}-c$ network of Wells for fixed axial ratio of 0.4 [13] (figs. 8).

We have shown that generalisations from bi- to tri-, quadra- and octa-continuous morphologies can be deduced from helical wrappings of sponges, derived from arrays of trees in the hyperbolic plane. The challenge is now to identify these structures in chemical systems. Multiple interpenetrating networks are well known in molecular crystals, and the chiral bicontinuous (slightly geometrically distorted) and quadra-continuous $\{10,3\}-a$ pattern have indeed been reported in a coordination polymeric molecular crystal [14]. The geometric regularity of these morphologies makes them promising candidates for polycontinuous mesophases in soft molecular systems containing three and four immiscible moieties. Further analysis must invoke a more concrete physical model for the self-assembly process, and will be done elsewhere. We note that these examples, that are the most symmetric decorations of the quasi-homogeneous sponges, are likely to be the most homogeneous partitions of space into multiple identical three-dimensional domains. These polycontinuous structures are thus the analogues of the energetically favoured bicontinuous forms, described by the $P, D$ and $G$ structures.

We thank C. OGUEY for discussions and advice. VRML images of the graphs can be found at www.mesoscale.anu.edu.au. We are grateful to D. Huson for his Funtiles software, used here to draw the underlying kaleidoscopic tilings of figs. 3 .

\section{REFERENCES}

[1] Scriven L. E., Nature, 263 (1976) 123.

[2] Luzzati V. and Spegt P. A., Nature, 215 (1967) 701.

[3] Larsson K., Fontell K. and Krog N., in Chem. Phys. Lipids, 27 (1980) 321.

[4] Hyde S. T., Andersson S., Larsson K., Blum Z., Landh T., Lidin S. and Ninham B. W., The Language of Shape (Elsevier, Amsterdam) (1997).

[5] Israelachvili J. N., Mitchell D. J. and Ninham B. W., J. Chem. Soc. Faraday Trans. 2, 72 (1976) 1525.

[6] Sadoc J.-F. and Charvolin J., J. Phys. (Paris), 47 (1986) 683.

[7] Hyde S. T., J. Phys. Chem., 93 (1989) 1458.

[8] Hyde S. T. and Oguey C., to be published in Eur. Phys. J. B.

[9] Conway J. H., in Groups, Combinatorics and Geometry (London Math. Soc. Lecture Note Series), Vol. 47 (1992) p. 438.

[10] Huson D. H., to be published in DIMACS Series in Discrete Mathematics and Theoretical Computer Science, Vol. 51, Discrete Mathematical Chemistry, edited by P. HANsen, P. Fowler and M. ZHENG.

[11] Sadoc J.-F. and Charvolin J., Acta Crystallogr. A, 45 (1989) 10.

[12] Fogden A. and Hyde S. T., Acta Crystallogr. A, 48 (1992) 442; Acta Crystallogr. A, 48 (1992) 575.

[13] Wells A. F., Three-dimensional Nets and Polyhedra (John Wiley and Sons, New York) (1977).

[14] Kepert, C.J. and Rosseinsky, M.J., Chem. Commun., (1998) 31. 